\title{
Differential Ghost Imaging
}

\author{
F. Ferri, ${ }^{*}$ D. Magatti, L. A. Lugiato, and A. Gatti \\ CNR-INFM-CNISM, Dipartimento di Fisica e Matematica, Università dell'Insubria, Via Valleggio 11, 22100 Como, Italy
}

(Received 5 February 2010; published 25 June 2010)

\begin{abstract}
We present a new technique, differential ghost imaging (DGI), which dramatically enhances the signalto-noise ratio (SNR) of imaging methods based on spatially correlated beams. DGI can measure the transmission function of an object in absolute units, with a SNR that can be orders of magnitude higher than the one achievable with the conventional ghost imaging (GI) analysis. This feature allows for the first time, to our knowledge, the imaging of weakly absorbing objects, which represents a breakthrough for GI applications. Theoretical analysis and experimental and numerical data assessing the performances of the technique are presented.
\end{abstract}

PACS numbers: $42.50 . \mathrm{Ar}$

Ghost imaging (GI) is a novel imaging technique which has become increasingly popular over the last decade, [117]. In GI the object image is retrieved by using two spatially correlated beams: the reference beam, which never interacts with the object and is measured with a pixelated detector, and the object beam, which, after illuminating the object is collected with a bucket detector, with no spatial resolution. By correlating the reference beam with the bucket signal, the "ghost" image is retrieved. The GI technique offers great potentialities with respect to standard imaging because it allows the imaging of objects located in optically harsh (difficult to be reached) or noisy environments, where standard techniques are likely to fail. Other potentialities are in the direction of compressive sensing [18], where the number of averages needed to recover an object may be lower than the number of pixels needed to resolve it.

In spite of these remarkable features, studies on GI have so far mainly focused on the fundamental debate of whether GI is an intrinsic quantum phenomenon requiring the entanglement of photon pairs, or it can be realized with classically correlated light beams [3-15]. This debate seems to have arrived at an end point where a substantial equivalence between the classical and quantum approaches was demonstrated [4-10,12,14,15], with some distinguishing features arising, e.g., in the signal-to-noise ratio (SNR) [17]. However, the possibility of implementing GI with classical sources still seems a pure academic achievement, because so far, with only few exceptions [16,18], only the imaging of simple binary objects (transmission 0 or 1 ) such as a double slit have been carried out. When it comes to real sensing and/or imaging applications, such as the imaging of a low contrast object or the location and possibly sizing of a small absorbing obstacle in a large beam, the SNR of conventional GI methods is remarkably low, and very long measurement times would be necessary $[17,18]$. As a consequence, the great potentiality offered by GI cannot be exploited.

In this Letter we focus on the thermal case, and we present a new method that we name differential ghost imaging (DGI) that overcomes this limitation and allows us to apply, for the first time to our knowledge, the GI protocol to realistic and potentially interesting problems. The technique can measure the transmission function of an object in absolute units, with a SNR that, depending on the object transmission relative variance, can be orders of magnitude higher than the one achievable with conventional GI. Thus DGI paves the way to real applications of the GI protocol, with the possibility of exploiting all the advantages of GI against standard imaging.

The optical setup is shown in Fig. 1 and is identical (except for the bucket detection, see below) to the one reported in Ref. [15]. The pseudothermal source operates at $\lambda=0.532 \mu \mathrm{m}$ and produces a collimated beam of deepFresnel speckles [19-21] whose size $\delta_{0} \sim 84.7 \mu \mathrm{m}$ does not change upon propagation. The area of the beam is $A_{\text {beam }} \sim 32.9 \mathrm{~mm}^{2}$ and contains $N_{\text {speckle }}=A_{\text {beam }} / A_{\text {coh }} \sim$ 4590 speckles $\left(A_{\text {coh }}=\delta_{0}^{2}\right)$. The speckle beam is divided by a cube beam splitter in two spatially correlated beams, the object and the reference beams, whose intensity distributions are $I_{1}\left(\mathbf{x}_{1}\right)$ and $I_{2}\left(\mathbf{x}_{2}\right)$, respectively. The object beam hits the object at a distance $z_{1}=130 \mathrm{~mm}$ from the source, and the bucket signal $S_{1}$ is collected with a photodiode. The reference beam $I_{2}\left(\mathbf{x}_{2}\right)$ is detected at the same distance $z_{1}=z_{2}$ with a CCD sensor whose square pixels have a size $6.67 \mu \mathrm{m} \ll \delta_{0}$. The reference bucket $S_{2}$ is obtained by summing the signals out from all the CCD pixels.

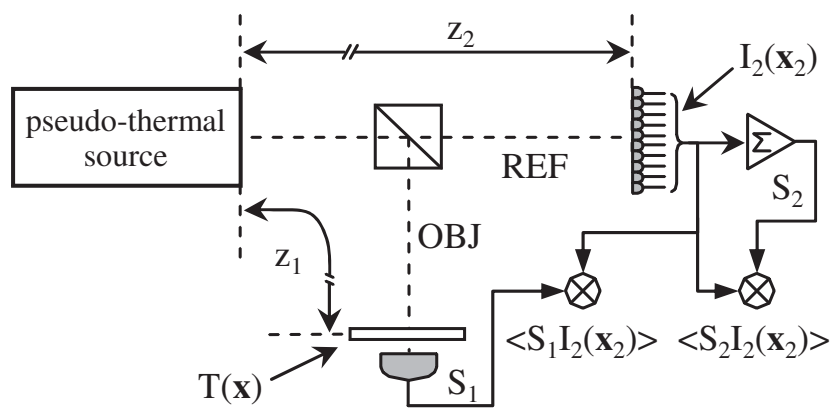

FIG. 1. Schematic diagram of the experimental setup. 
In the following we shall adopt a classical formalism, in which all the detected photon numbers are so large that the shot noise is always negligible. Thus, the reference and object beams are perfectly correlated stochastic variables, and we can set $I_{1}\left(\mathbf{x}_{1}\right)=\alpha I_{2}\left(\mathbf{x}_{2}\right)$, where $\alpha$ is a factor which takes into account any unbalancing (beam splitter and detectors) between the two arms.

In the conventional GI analysis the ghost image is retrieved by correlating $I_{2}\left(x_{2}\right)$ with the bucket signal $S_{1}$

$$
S_{1}=\int_{A_{\text {beam }}} I_{1}\left(\mathbf{x}_{1}\right) T\left(\mathbf{x}_{1}\right) d^{2} \mathbf{x}_{1}
$$

where $T(\mathbf{x})$ denotes the (intensity) transmission function of the object. The measured quantity in GI is [5]:

$$
\left\langle O\left(\mathbf{x}_{2}\right)\right\rangle=\left\langle\delta S_{1} \delta I_{2}\left(\mathbf{x}_{2}\right)\right\rangle
$$

where $\langle\cdots\rangle$ is an average over independent speckle configurations and $\delta S_{1}=S_{1}-\left\langle S_{1}\right\rangle, \quad \delta I_{2}\left(\mathbf{x}_{2}\right)=I_{2}\left(\mathbf{x}_{2}\right)-$ $\left\langle I_{2}\left(\mathbf{x}_{2}\right)\right\rangle$. Note that the procedure defined by Eq. (2) is equivalent to the removal of the dc photocurrent component done in [11,13]. Equation (2) can be worked out in terms of $T\left(\mathbf{x}_{2}\right)$ obtaining $[5,15]$

$$
\begin{aligned}
\left\langle O\left(\mathbf{x}_{2}\right)\right\rangle & =A_{\mathrm{coh}}\left\langle I_{1}\left(\mathbf{x}_{2}\right)\right\rangle\left\langle I_{2}\left(\mathbf{x}_{2}\right)\right\rangle[T \otimes \gamma]\left(\mathbf{x}_{2}\right) \\
& \approx A_{\mathrm{coh}}\left\langle I_{1}\left(\mathbf{x}_{2}\right)\right\rangle\left\langle I_{2}\left(\mathbf{x}_{2}\right)\right\rangle T\left(\mathbf{x}_{2}\right)
\end{aligned}
$$

where $A_{\text {coh }}$ is the speckle coherence area defined in terms of the complex coherence factor $\mu(\mathbf{x})$ [22] as $A_{\text {coh }}=$ $\int|\mu(\mathbf{x})|^{2} d^{2} \mathbf{x}$. The symbol $\otimes$ denotes convolution between $T(\mathbf{x})$ and the function $\gamma(\mathbf{x})=|\mu(\mathbf{x})|^{2} / A_{\text {coh }}$, which represents the point spread function (PSF) of the technique ( $\left.\int \gamma(\mathbf{x}) d^{2} \mathbf{x}=1\right)$. The second line of Eq. (3) is valid under the assumption of "perfect resolution", i.e., when the linear width $\delta_{0} \sim \sqrt{A_{\text {coh }}}$ of $\gamma(\mathbf{x})$ is much smaller than the length scales characterizing the variations of $T(\mathbf{x})$. Since all the factors in front of $T(\mathbf{x})$ are accurately measurable, Eq. (3) provides, in principle, a way of measuring $T(\mathbf{x})$ in absolute units. However, as reported in [23], the presence of extra sources of noise (as fluctuations in the emitting power of the thermal source), introduces a spurious offset in $T(\mathbf{x})$, which prevents from retrieving it in absolute units. The noise associated to the stochastic variable $O\left(\mathbf{x}_{2}\right)$ is

$$
\left\langle\delta O^{2}\left(\mathbf{x}_{2}\right)\right\rangle=\left\langle\delta S_{1}^{2} \delta I_{2}^{2}\left(\mathbf{x}_{2}\right)\right\rangle-\left\langle\delta S_{1} \delta I_{2}\left(\mathbf{x}_{2}\right)\right\rangle^{2}
$$

whose exact computation is rather cumbersome because it involves a series of moments up to the order 4 in the intensities [17,23]. However, for Gaussian speckle fields, it is possible to show [23] that the dominant term is given by the "uncorrelated" contribution

$$
\left\langle\delta O^{2}\left(\mathbf{x}_{2}\right)\right\rangle=\left\langle\delta S_{1}^{2}\right\rangle\left\langle\delta I_{2}^{2}\left(\mathbf{x}_{2}\right)\right\rangle .
$$

To extract the dependence of Eq. (5) on $T(\mathbf{x})$, we can make the simplifying assumption of uniform illumination, i.e., that the average speckle beams are constant over their cross area $\left[\left\langle I_{1}\left(\mathbf{x}_{1}\right)\right\rangle=\left\langle I_{1}\right\rangle,\left\langle I_{2}\left(\mathbf{x}_{2}\right)\right\rangle=\left\langle I_{2}\right\rangle\right]$. By assuming again perfect resolution $(T \otimes \gamma \sim \gamma)$, after some calculations, we get to the result [23]

$$
\left\langle O^{2}\right\rangle=A_{\text {coh }} A_{\text {beam }}\left\langle I_{1}\right\rangle^{2}\left\langle I_{2}\right\rangle^{2} \overline{T^{2}}
$$

where $\quad \overline{T^{2}}=\int_{A_{\text {beam }}}\langle I(\mathbf{x})\rangle T^{2}(\mathbf{x}) d^{2} \mathbf{x} / \int_{A_{\text {beam }}}\langle I(\mathbf{x})\rangle d^{2} \mathbf{x} \approx$ $\int_{A_{\text {beam }}} T^{2}(\mathbf{x}) d^{2} x / A_{\text {beam }}$ is the average quadratic transmission function of the object. If we define the minimum variation of $\Delta\left\langle O\left(\mathbf{x}_{2}\right)\right\rangle$ as the signal that needs to be detected, and average over $m$ independent measurements, we obtain from Eqs. (3) and (6) that the SNR of the conventional GI analysis is

$$
(\mathrm{SNR})_{\mathrm{GI}}=\frac{\left[\Delta\left\langle O\left(\mathbf{x}_{2}\right)\right\rangle\right]_{\mathrm{min}}^{2}}{\left\langle\Delta O^{2}\right\rangle}=\frac{m}{N_{\text {speckle }}} \frac{\Delta T_{\min }^{2}}{\overline{T^{2}}}
$$

where $N_{\text {speckle }}=A_{\text {beam }} / A_{\text {coh }}$ is the number of speckles in the beam and $\Delta T_{\min }$ is the minimum variation of the object transmission function to be detected. Equation (7) shows not only the well-known result [17] that $(\mathrm{SNR})_{\mathrm{GI}}$ scales as $1 / N_{\text {speckle }}$, but also as $1 / \overline{T^{2}}$. Thus, quasitransparent objects $\left(\overline{T^{2}} \sim 1\right)$ are much more difficult to retrieve than highly absorbing objects $\left(\overline{T^{2}} \ll 1\right)$ and may require an exceedingly high number of measurements.

To implement the ghost imaging technique on quasitransparent objects, we developed a new method that we name differential ghost imaging (DGI). The idea is to improve the sensitivity of GI by using a new differential bucket signal $S_{-}$, which is sensitive only to the fluctuating part $\delta T(\mathbf{x})=T(\mathbf{x})-\bar{T}$. Thus

$$
S_{-}=\int_{A_{\text {beam }}} I_{1}\left(\mathbf{x}_{1}\right) \delta T\left(\mathbf{x}_{1}\right) d^{2} \mathbf{x}_{1}
$$

where $\bar{T}=\int_{A_{\text {beam }}}\langle I(\mathbf{x})\rangle T(\mathbf{x}) d^{2} \mathbf{x} / \int_{A_{\text {beam }}}\langle I(\mathbf{x})\rangle T(\mathbf{x}) d^{2} x$ is the average transmission function of the object. To find an operative procedure for measuring $S_{-}$, we define the reference bucket signal $S_{2}$

$$
S_{2}=\int_{A_{\text {beam }}} I_{2}\left(\mathbf{x}_{2}\right) d^{2} \mathbf{x}_{2}
$$

and notice that $\bar{T}$ can be expressed in terms of $\left\langle S_{2}\right\rangle$ as

$$
\bar{T}=\frac{1}{\alpha} \frac{\left\langle S_{1}\right\rangle}{\left\langle S_{2}\right\rangle} .
$$

Thus $S_{-}$can be put in the operative form

$$
S_{-}=S_{1}-\frac{\left\langle S_{1}\right\rangle}{\left\langle S_{2}\right\rangle} S_{2}
$$

which makes it easily measurable. The DGI technique works by substituting $S_{1}$ with $S_{-}$and performing the standard correlation analysis [Eq. (2)]. Therefore, the quantity measured in DGI is

$$
\begin{aligned}
\left\langle O_{-}\left(\mathbf{x}_{2}\right)\right\rangle & =\left\langle\delta S_{-} \delta I_{2}\left(\mathbf{x}_{2}\right)\right\rangle \\
& =\left\langle S_{1} I_{2}\left(\mathbf{x}_{2}\right)\right\rangle-\frac{\left\langle S_{1}\right\rangle}{\left\langle S_{2}\right\rangle}\left\langle S_{1} I_{2}\left(\mathbf{x}_{2}\right)\right\rangle
\end{aligned}
$$

where we used Eq. (11) and the property $\left\langle S_{-}\right\rangle=0$. 
Equation (12) can be worked out in terms of $\delta T\left(\mathbf{x}_{2}\right)$ and, under the same assumptions used for Eq. (3), it becomes

$$
\begin{aligned}
\left\langle O_{-}\left(\mathbf{x}_{2}\right)\right\rangle & =A_{\text {coh }}\left\langle I_{1}\left(\mathbf{x}_{2}\right)\right\rangle\left\langle I_{2}\left(\mathbf{x}_{2}\right)\right\rangle[\delta T \otimes \gamma]\left(\mathbf{x}_{2}\right) \\
& \approx A_{\text {coh }}\left\langle I_{1}\left(\mathbf{x}_{2}\right)\right\rangle\left\langle I_{2}\left(\mathbf{x}_{2}\right)\right\rangle \delta T\left(\mathbf{x}_{2}\right)
\end{aligned}
$$

which is identical to Eq. (3), but with $\delta T\left(\mathbf{x}_{2}\right)$ instead of $T\left(\mathbf{x}_{2}\right)$. Thus DGI works by retrieving the fluctuating part $\delta T(\mathbf{x})$ and, by combining Eq. (13) with Eq. (10), the entire object transmission function $T(\mathbf{x})$ can be recovered. We emphasize that, differently from what happens in GI, DGI is immune from extra sources of noise [23], so that it effectively allows us to retrieve $T(\mathbf{x})$ in absolute units. The noise associated to the variable $O_{-}\left(\mathbf{x}_{2}\right)$ is

$$
\left\langle\delta O_{-}^{2}\left(\mathbf{x}_{2}\right)\right\rangle=\left\langle\delta S_{-}^{2} \delta I_{2}^{2}\left(\mathbf{x}_{2}\right)\right\rangle-\left\langle\delta S_{-} \delta I_{2}\left(\mathbf{x}_{2}\right)\right\rangle^{2}
$$

whose computation can be carried out as for Eq. (4), obtaining, under the same assumptions,

$$
\left\langle O_{-}^{2}\right\rangle=A_{\text {coh }} A_{\text {beam }}\left\langle I_{1}\right\rangle^{2}\left\langle I_{2}\right\rangle^{2} \overline{\delta T^{2}}
$$

where $\overline{\delta T^{2}}=\overline{T^{2}}-(\bar{T})^{2}$ is the variance of $T(\mathbf{x})$. From Eqs. (13) and (15) we arrive at the final result for the SNR after $m$ independent measurements

$$
(\mathrm{SNR})_{\mathrm{DGI}}=\frac{\left[\Delta\left\langle O_{-}\left(\mathbf{x}_{2}\right)\right\rangle\right]_{\mathrm{min}}^{2}}{\left\langle\Delta O_{-}^{2}\right\rangle}=\frac{m}{N_{\text {speckle }}} \frac{\Delta T_{\mathrm{min}}^{2}}{\overline{\delta T^{2}}}
$$

which is identical to Eq. (7), but with $\overline{\delta T^{2}}$ in place of $\overline{T^{2}}$. The ratio between Eqs. (16) and (7)

$$
\frac{(\mathrm{SNR})_{\mathrm{DGI}}}{(\mathrm{SNR})_{\mathrm{GI}}}=1+\frac{1}{\sigma_{\mathrm{rel}}^{2}}
$$

is always $>1$ and shows that the enhancement of the SNR between the two techniques depends only on the object relative variance $\sigma_{\text {rel }}^{2}=\overline{\delta T^{2}} /(\bar{T})^{2}$. Thus, for highly transparent objects $\left[\overline{\delta T^{2}} \ll(T)^{2}, \sigma_{\text {rel }}^{2} \ll 1\right]$, the DGI technique works much better than the conventional one. Conversely, for highly absorbing objects $\left[(T)^{2} \ll \overline{\delta T^{2}} \ll 1, \sigma_{\text {rel }}^{2} \rightarrow \infty\right]$ the two techniques perform quite similarly.

The first experiment shows the striking differences between conventional GI and DGI when a small object in a large beam has to be detected and sized. Two totally $(T=$ 0 ) absorbing particles, 820 and $400 \mu \mathrm{m}$ in diameter, were glued on a microscope slide $(T=0.92)$ and shined with a beam of size $\sim 5.7 \mathrm{~mm}$. Thus for this object $(\bar{T} \sim 0.90$ and $\sigma_{\text {rel }} \sim 0.14$ ) the SNR enhancement predicted by Eq. (17) is $(\mathrm{SNR})_{\mathrm{DGI}} /(\mathrm{SNR})_{\mathrm{GI}} \sim 52$. The GI and DGI analyses were performed on the same raw data, and the images retrieved after $m=10^{4}$ averages are reported in Fig. 2. While the GI image is almost completely blurred, the DGI image is neatly resolved, allowing the retrieval of both particle sizes and positions. The measured enhancement of the SNR was $\sim 66$, higher than expected (see below).

The second experiment checked the validity of Eqs. (7) and (16). We used a binary ( $T=0$ or $T=1)$ object, made by a dark knife obstructing the beam, so that the ratio between the obstacle area and the beam size $\epsilon=$
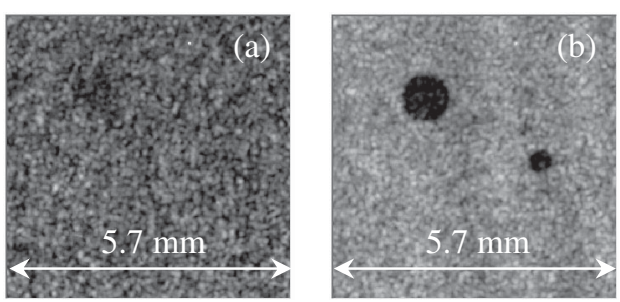

FIG. 2. Conventional GI (a) and DGI (b) of two totally absorbing particles 820 and $400 \mu \mathrm{m}$ in diameter, retrieved after $m=$ $10^{4}$ averages. The number of speckles in the beam was $N_{\text {speckle }} \sim$ 4590 .

$A_{\text {obst }} / A_{\text {beam }}$ could be varied. For this object $\bar{T}=1-\epsilon$, $\overline{T^{2}}=\bar{T}, \overline{\delta T^{2}}=\bar{T}(1-\bar{T})$. Figure 3 compares $(\mathrm{SNR})_{\mathrm{DGI}}$ and $(\mathrm{SNR})_{\mathrm{GI}}$ normalized to $m \Delta T_{\min }^{2}$ as a function of $\epsilon$ (bottom scale) and $\bar{T}$ (top scale). The experimental data for DGI (solid circles) and for GI (solid squares) are plotted against theory (solid lines). The open symbols are obtained from computer simulations in which $\sim 2 \%$ rms fluctuations of the power emitted by the thermal source was introduced. The crosses indicate the SNR's associated to the data of Fig. 2. Beside demonstrating the excellent agreement between DGI data and theory, Fig. 3 shows the striking difference between the two techniques: they behave quite similarly when the object is a small aperture $(\epsilon \rightarrow 1)$, but if the object is a small obstacle $(\epsilon \rightarrow 0)$, GI becomes highly inefficient while DGI remains quite performing, being $(\mathrm{SNR})_{\mathrm{DGI}} /(\mathrm{SNR})_{\mathrm{DGI}}=1 / \epsilon$. Furthermore, for conventional GI, the SNR retrieved from data and simulations is systematically smaller than the expected one. This discrepancy is due to the presence of other sources of noise, which degrade $(\mathrm{SNR})_{\mathrm{GI}}$ but not $(\mathrm{SNR})_{\mathrm{DGI}}$ [23].

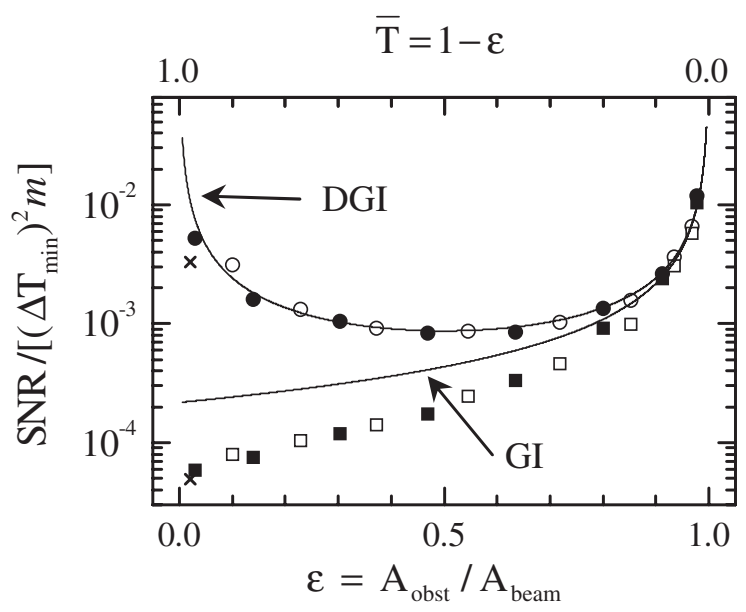

FIG. 3. Comparison between normalized SNR's of GI and DGI techniques for an obstacle object as a function of relative size $\epsilon=A_{\text {obst }} / A_{\text {beam }}$ and $\bar{T}$ (top horizontal axis). Theoretical behaviors [Eqs. (7) and (16)] are indicated by the two lines. The experimental data (solid symbols) and numerical simulations (open symbols), match accurately the theory only for DGI. Crosses indicate SNR values for the object of Fig. 2. 

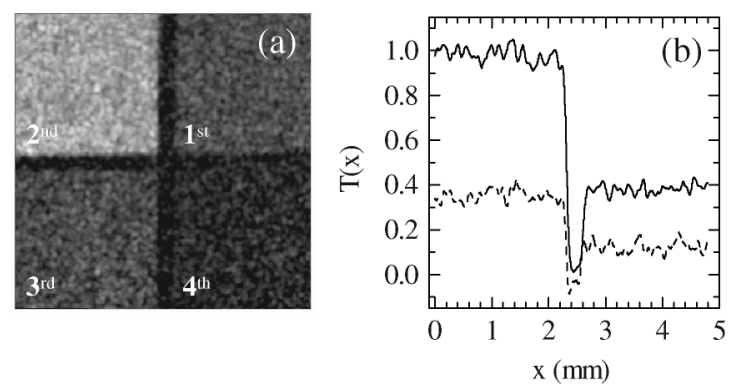

FIG. 4. Panel (a): DGI of an object made of two absorption filters partially staggered together, so to create four regions of transmission $T(\mathbf{x})=0.16$ (4th quadrant), 0.40 (1st and 3rd quadrants), 1.0 (2nd quadrant). Panel (b): averaged horizontal sections of the 2nd and 1st (solid line) and 3rd and 4th (dashed line) quadrants. Absolute accuracies were 0.04, number of averages $m=4 \times 10^{4}$.

The accuracy of the DGI technique in retrieving absolute values of $T(\mathbf{x})$ was ascertained by using an object made of two absorption filters $(T=0.40)$ partially staggered together. Thus in the region where the filters are overlapped $T(\mathbf{x})=0.16$, where they are not overlapped $T(\mathbf{x})=0.40$, and where there is no filter, $T(\mathbf{x})=1$. The DGI image retrieved after $m=4 \times 10^{4}$ averages is shown in Fig. 4(a), where the four regions are clearly recognizable. The absolute values of $T(\mathbf{x})$ are shown in Fig. 4(b), where the averaged horizontal sections of the 2nd and 1st (solid line) and 3rd and 4th (dashed line) quadrants are reported. The absolute accuracies in all of the four regions were $\sim 0.04$. When the same raw data of Fig. 4 were analyzed with the GI technique, the retrieved image was qualitatively similar to Fig. 4(a), but $T(\mathbf{x})$ was displaced by an offset of the same order of $\bar{T}$ (data not shown).

Finally, we tested the DGI technique with a continuous varying grayscale object, i.e., a slide representing a detail of the famous painting "Nascita di Venere" by S. Botticelli (Firenze, Italy, A.D. 1484). Figure 5(a) shows the image of the original slide after convolution with the PSF corresponding to a speckle size of $\delta_{0} \sim 85 \mu \mathrm{m}$. For this object $\bar{T} \sim 0.086$ and $\sigma_{\text {rel }} \sim 0.72$, so that the expected ratio $(\mathrm{SNR})_{\mathrm{DGI}} /(\mathrm{SNR})_{\mathrm{GI}} \sim 2.9$. Figures $5(\mathrm{~b})(\mathrm{DGI})$ and $5(\mathrm{c})$ (GI) show that the images retrieved $\left(m=10^{5}\right)$ with the two techniques are remarkably different with a SNR en-
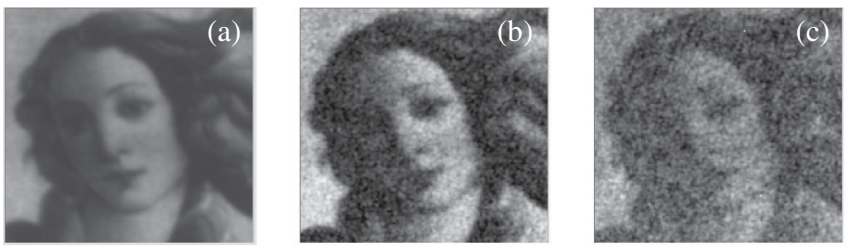

FIG. 5. Original slide (a),DGI (b) and GI (c) images of a detail of the famous painting "Nascita di Venere" by S. Botticelli (Firenze, Italy, A.D. 1484) retrieved after $m=10^{5}$ averages. The original image was convolved with a PSF of width equal to the speckle size $\delta_{0} \sim 85 \mu \mathrm{m}$. hancement of $\sim 6.4$, higher than expected. Once again the comparison between the DGI and GI techniques is better than what is predicted by Eq. (17), because DGI is more robust against extra source of noise.

In conclusion, we have presented a new technique called differential ghost imaging (DGI) that enhances dramatically the SNR of the GI protocol when applied to realistic complex objects, which are of interest in real word applications. We believe that this new technique will pave the way to the use of GI in many sensing or imaging problems, and in particular, in all the situations where standard techniques appear not to be adequate, such as when the object to be imaged is located in optically harsh or noisy environments.

We thank M. Molteni for the critical reading of the manuscript.

*Corresponding author; fabio.ferri@uninsubria.it

[1] T. B. Pittman, Y.H. Shih, D. V. Strekalov, and A. V. Sergienko, Phys. Rev. A 52, R3429 (1995).

[2] A. F. Abouraddy et al., Phys. Rev. Lett. 87, 123602 (2001); 93, 213903 (2004).

[3] R. S. Bennink, S. J. Bentley, R. W. Boyd, and J. C. Howell, Phys. Rev. Lett. 92, 033601 (2004).

[4] R. S. Bennink, S. J. Bentley, and R. W. Boyd, Phys. Rev. Lett. 89, 113601 (2002).

[5] A. Gatti, E. Brambilla, M. Bache, and L. A. Lugiato, Phys. Rev. Lett. 93, 093602 (2004).

[6] F. Ferri et al., Phys. Rev. Lett. 94, 183602 (2005).

[7] A. Valencia, G. Scarcelli, M. D'Angelo, and Y. Shih, Phys. Rev. Lett. 94, 063601 (2005).

[8] M. Bache et al., Phys. Rev. A 73, 053802 (2006).

[9] A. Gatti et al., J. Mod. Opt. 53, 739 (2006).

[10] J. H. Shapiro, Phys. Rev. A 78, 061802(R) (2008).

[11] G. Scarcelli, V. Berardi, and Y. Shih, Phys. Rev. Lett. 96, 063602 (2006).

[12] A. Gatti et al., Phys. Rev. Lett. 98, 039301 (2007).

[13] G. Scarcelli, V. Berardi, and Y. Shih, Appl. Phys. Lett. 88, 061106 (2006).

[14] L. Basano and P. Ottonello, Appl. Phys. Lett. 89, 091109 (2006).

[15] F. Ferri, D. Magatti, V. G. Sala, and A. Gatti, Appl. Phys. Lett. 92, 261109 (2008).

[16] R. Meyers, K. S. Deacon, and Y. Shih, Phys. Rev. A 77, 041801(R) (2008).

[17] B. I. Erkmen and J. H. Shapiro, Phys. Rev. A 79, 023833 (2009).

[18] O. Katz, Y. Bromberg, and Y. Silberberg, Appl. Phys. Lett. 95, 131110 (2009).

[19] R. Cerbino, Phys. Rev. A 75, 053815 (2007).

[20] A. Gatti D. Magatti, and F. Ferri, Phys. Rev. A 78, 063806 (2008).

[21] D. Magatti, A. Gatti, and F. Ferri, Phys. Rev. A 79, 053831 (2009).

[22] J. W. Goodman, Speckle Phenomena in Optics (RobertsCompany, Greenwood Village, CO, 2006), pp. 90-92.

[23] A. Gatti et al. (to be published). 